\title{
Nitidine chloride possesses anticancer property in lung cancer cells through activating Hippo signaling pathway
}

Jing Zhang ${ }^{1}$, Linhui $\mathrm{Wu}^{2}$, Chaoqun Lian ${ }^{3}$, Shuo Lian ${ }^{4}$, Shimeng Bao ${ }^{5}$, Jisheng Zhang ${ }^{6}$, Peter Wang $\mathbb{1}^{3}$, Jia Ma ${ }^{3}$ and Yuyun $\mathrm{Li}^{7}$

\begin{abstract}
Nitidine chloride (NC) has significant anti-tumor properties; however, the precise mechanism related to NC still needs further investigation. This study intends to investigate the anti-tumor functions and the feasible molecular basis of NC in NSCLC cells. Therefore, we determined the mechanism of NC-mediated anti-tumor function through various methods. Cell proliferation ability and migration and invasion were detected by CCK-8, colony formation assay and Transwell assay, respectively. Furthermore, flow cytometry was used to detect apoptosis, cell cycle and ROS. Moreover, protein expression level was measured by western blot. Our results showed that NC can inhibit the growth, motility of NSCLC cells, induce apoptosis and arrest cell cycle. Meanwhile, NC increased the level of ROS in NSCLC cells. Moreover, western blot data showed that NC suppressed the expression of Lats1, Mob1, and YAP, and enhanced the expression of p-Lats1, p-Mob1, p-YAP1 (ser127). Overall, our research reveals that NC exerts anticancer activity by activating and modulating the Hippo signaling pathway.
\end{abstract}

\section{Introduction}

Non-small cell lung cancer (NSCLC) is a malignant tumor that poses a serious threat to human health ${ }^{1}$. The treatment of NSCLC patients is mainly based on postoperative chemotherapy combined with radiotherapy ${ }^{2}$, but the side effects of radiotherapy and chemotherapy are very conspicuous, and patients will eventually develop drug resistance leading to treatment failure ${ }^{3}$. Thus, it is necessary to find key target molecules and new therapeutic strategies to inhibit the progress of NSCLC.

In recent years, the newly discovered Hippo signaling pathway has gradually received extensive attention.

\footnotetext{
Correspondence: Jia Ma (majiamj10@126.com) or Yuyun Li (bbmcliyuyun@163. com)

${ }^{1}$ Department of Genetics, School of Life Sciences, Bengbu Medical College, Anhui 233030, China

${ }^{2}$ Bengbu Medical College Key Laboratory of Cancer Research and Clinical Laboratory Diagnosis, Bengbu Medical College, Anhui 233030, China

Full list of author information is available at the end of the article

These authors contributed equally: Jing Zhang, Linhui Wu

Edited by Alessandro Rufini
}

Studies have shown that its abnormalities are often involved in the occurrence and development of human tumors. A series of conserved protein kinases constitute the core components of Hippo signaling pathway. In mammals, Hippo pathway includes mammalian Sterile 20-like kinase 1 and 2 (MST1/2), large tumor suppressor kinase 1 and 2 (LATS1/2), and Mps one binder protein 1 (MOB1), Yes-associated protein (YAP), and transcriptional coactivator with PDZ-binding motif (TAZ) $)^{4,5}$. When the growth inhibitory signal is transmitted to the cell membrane, the Hippo signal is activated, then MST1/ 2 and WW45 phosphorylate and activate the downstream LATS1/2 kinase and adaptor protein MOB1, and then phosphorylate the downstream transcriptional regulator YAP/TAZ. As an oncogene, YAP/TAZ promotes excessive cell proliferation and counteracts apoptosis ${ }^{6}$. Phosphorylated YAP/TAZ binds to 14-3-3 in the cytoplasm and gradually degrades and ubiquitinates, thus failing to enter nuclear transcription to initiate target gene expression, and ultimately inhibits organ size, tumor cell 


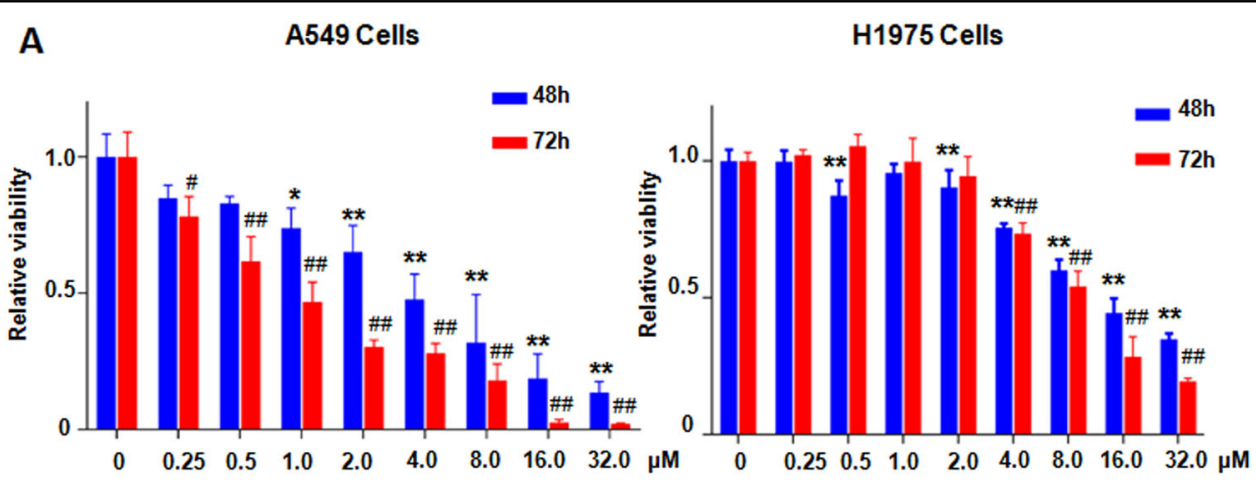

B
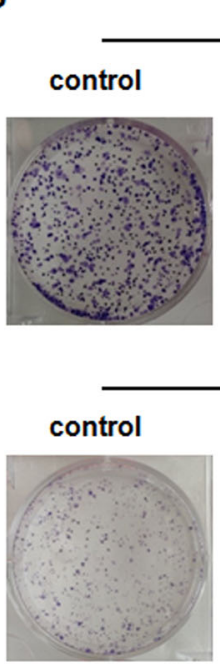

A549 Cells

\section{$4 \mu \mathrm{M}$}

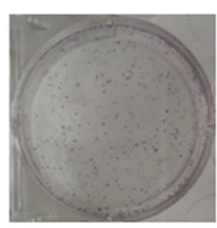

H1975 Cells

$14 \mu \mathrm{M}$

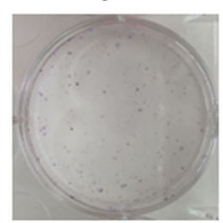

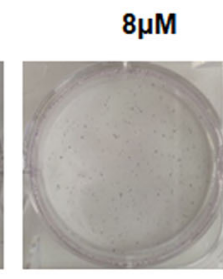

$32 \mu \mathrm{M}$

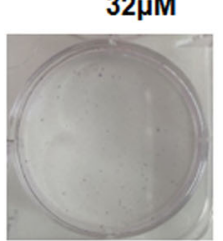

C
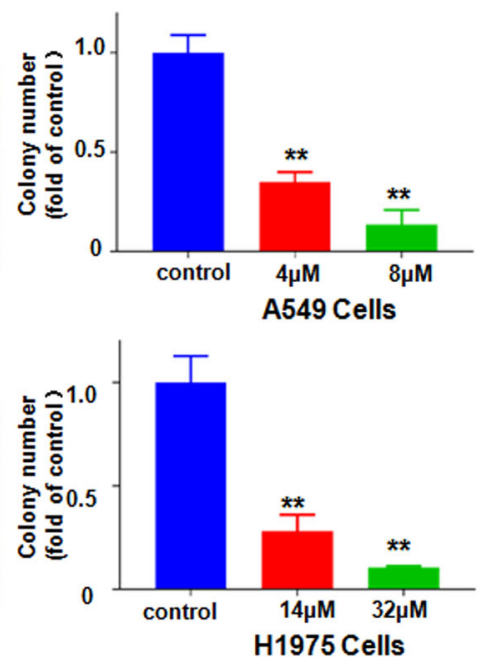

Fig. 1 NC restrained viability of NSCLC cells. a The viability of A549 and H1975 cells was detected by CCK-8 assay after treatment with various concentrations of $\mathrm{NC}$ for $48 \mathrm{~h}$ and $72 \mathrm{~h}$. ${ }^{*} P<0.05$, ${ }^{* *} P<0.01$ vs. control $\left(48 \mathrm{~h}\right.$ ); ${ }^{*} P<0.05$, ${ }^{\# \#} P<0.01$ vs. control $(72 \mathrm{~h}$ ). b Colony formation assay was performed in A549 and $\mathrm{H} 1975$ cells treated with NC at the indicated concentration for 48 h. c Quantification of the colony number in (b). ${ }^{* *} P<0.01$ vs. control, the same in the following figure legends.

proliferation, and metastasis ${ }^{5,7}$. The Hippo pathway is very conservative during evolution, and it is crucial in regulating cell proliferation, differentiation, tissue regeneration, organ size, as well as cancer development ${ }^{8-10}$. In a variety of human tumors, Hippo signaling pathway is downregulated, suggesting that it is closely related to tumorigenesis ${ }^{11,12}$. Evidence also indicates that Hippo signaling pathway plays a key role in the development of $\mathrm{NSCLC}^{4}$. Consequently, it is very important to develop new anti-cancer therapies through targeting Hippo pathway.

Nitidine chloride $(\mathrm{NC})$ is a pure natural alkaloid with biological activity ${ }^{13}$. Previous studies revealed that $\mathrm{NC}$ has multiple pharmacological functions, such as anti-fungal, anti-inflammatory, and anti-oxidant properties ${ }^{14,15}$. Recently, several studies revealed tumor-suppressive functions of $\mathrm{NC}$ in various types of human malignant cancers $^{16-19}$. As a natural anti-cancer drug, NC has received extensive attention. We previously demonstrated that NC had an inhibitory effect on prostate cancer cells and osteosarcoma cells ${ }^{20,21}$. However, the role of $\mathrm{NC}$ in human lung cancer cells and its potential molecular mechanism need to be further elucidated. In this study, we sought to investigate the role of NC in inhibiting the biological function of NSCLC cells. Moreover, whether its molecular mechanism is related to the Hippo pathway was further explored.

\section{Results}

\section{NC inhibited cell viability and colony formation}

CCK8 assays and colony formation assays were performed in NSCLC cells. In CCK8 assays, A549 and H1975 cells were incubated in different concentrations of $\mathrm{NC}(0.25,0.5,1,2,4,8,16,32 \mu \mathrm{M})$ for 48 and $72 \mathrm{~h}$, 
respectively. The results showed that NC significantly inhibited the viability of NSCLC cells compared to the control group with $0.1 \%$ DMSO alone, and this effect was more pronounced with increasing doses and time (Fig. 1a). Almost 50\% cell viability in A549 and H1975 cells was suppressed by $4 \mu \mathrm{M}$ and $14 \mu \mathrm{M}$ NC after $48 \mathrm{~h}$ treatment, respectively. NC induced about $70 \%$ inhibition of cell viability in A549 cells and H1975 cells treated with $8 \mu \mathrm{M}$ and $32 \mu \mathrm{M}$ for $48 \mathrm{~h}$, respectively. Therefore, we chose $4 \mu \mathrm{M}$ and $8 \mu \mathrm{M} \mathrm{NC}$ for A549 cells and $14 \mu \mathrm{M}$ and $32 \mu \mathrm{M} \mathrm{NC}$ for $\mathrm{H} 1975$ cells to conduct subsequent experiments. Next, the effect of NC treatment on the number of cell-forming clones was further examined by colony formation assay. The results showed that as the concentration increased, NC significantly reduced the number of colonies in A549 and H1975 cells (Fig. 1b, c). Altogether, NC effectively suppressed cell viability in NSCLC cells.

\section{NC inhibited motility of NSCLC cells}

Transwell assay further dissects the effects of $\mathrm{NC}$ on motility ofA549 and H1975 cells in vitro. As shown in Fig. $2 \mathrm{a}, \mathrm{b}$, the results from transwell showed that as the NC concentrations were increased, A549 or H1975 cells that migrated and invaded into the lower chamber became less. Due to that NC did not inhibit cell viability in A549 and H1975 cells at $24 \mathrm{~h}$ when these cells were cultured without FBS (data not shown), our results revealed that NC inhibited motility of NSCLC cells.

\section{NC induced cell apoptosis}

Annexin V-FITC/PI double fluorescence staining and flow cytometry were used to detect apoptosis in NSCLC cells treated with $\mathrm{NC}$ for $48 \mathrm{~h}$. The results showed that NC treatment enhanced cell apoptosis. The apoptosis rates of A549 cells were increased from $12.58 \pm 2.20 \%$ of the control group to $45.97 \pm 2.75 \%$ and $60.73 \pm 5.05 \%$

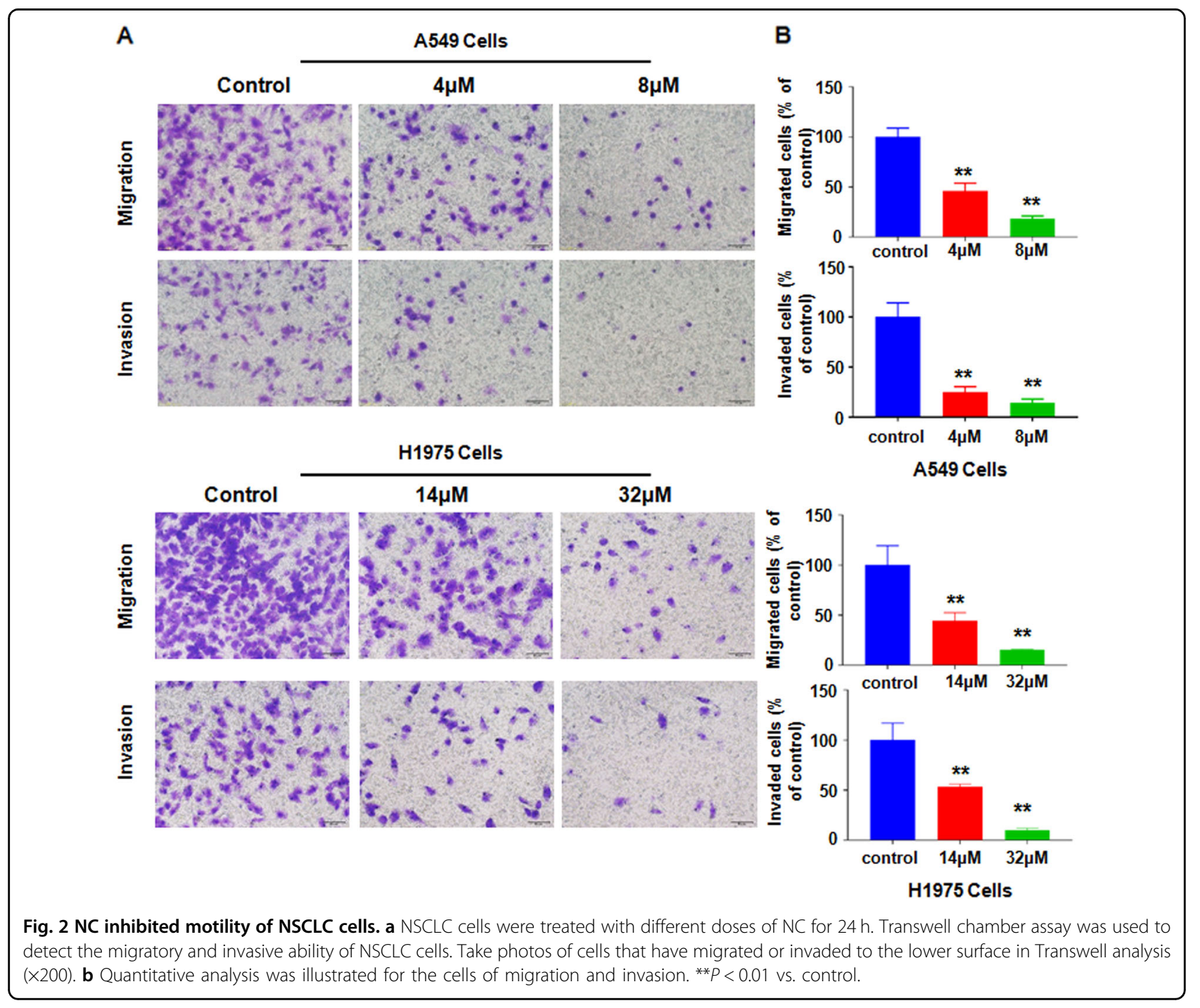



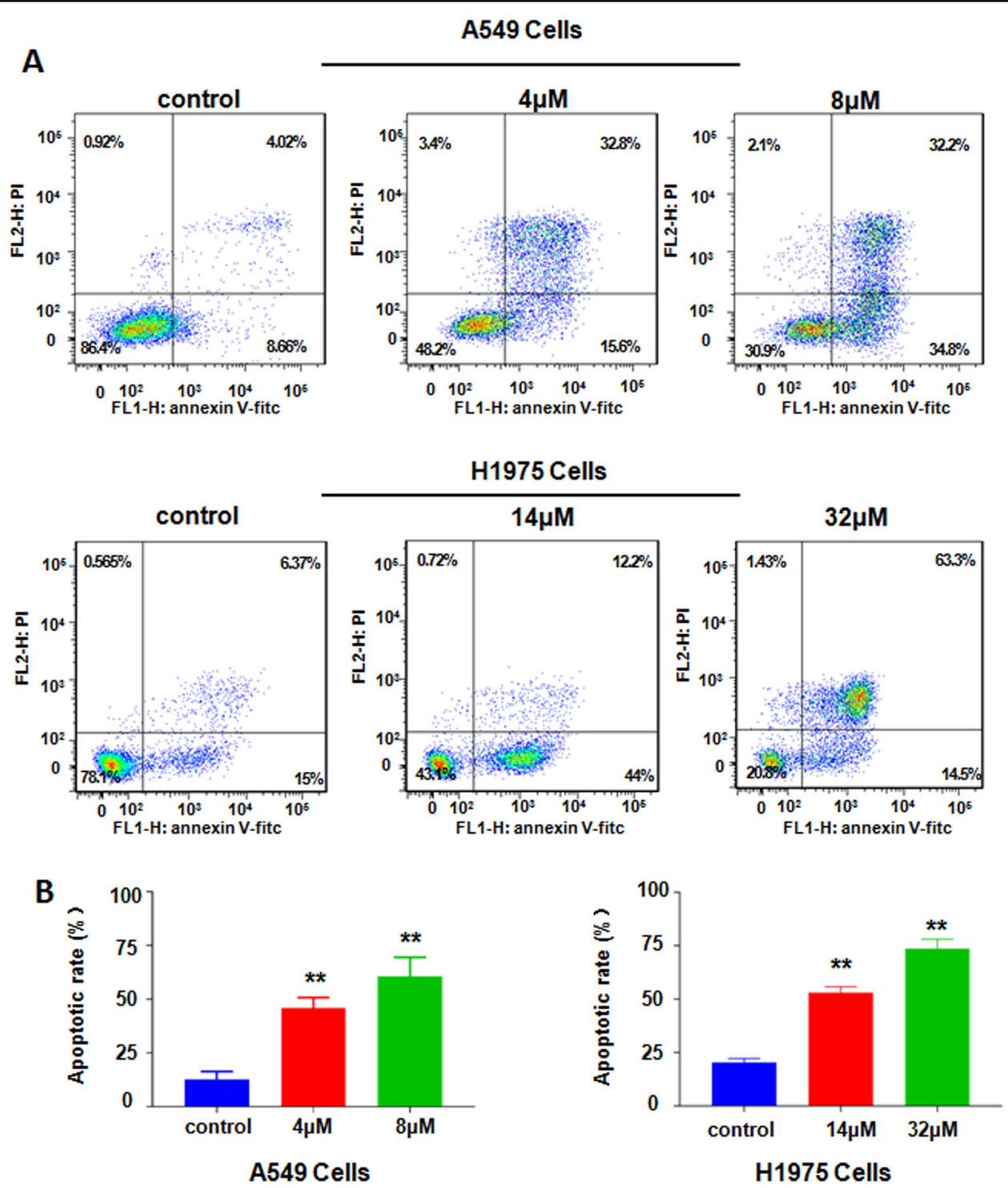

Fig. 3 NC induced apoptosis in NSCLC cells. a Flow cytometry analysis of cell apoptosis in A549 and H1975 cells was determined after treating with different doses of NC for $48 \mathrm{~h}$. b Quantitative analysis of percentages of apoptotic cells in the A549 and H1975 cells. ${ }^{* *} P<0.01$ vs. control.

following exposure to $\mathrm{NC}(4$ and $8 \mu \mathrm{M})$, respectively (Fig. $3 \mathrm{a}, \mathrm{b})$. And treatment with $\mathrm{NC}(14$ and $32 \mu \mathrm{M})$ in $\mathrm{H} 1975$ cells caused $53.07 \pm 1.59 \%$ and $73.6 \pm 2.55 \%$ apoptosis rate (Fig. 3a, b). The results dissected that NC significantly stimulated NSCLC cell apoptosis.

\section{NC caused cell cycle arrest}

Through the detection by flow cytometry, we could acquire the distribution of cell cycle at each phase. Compared to control, NSCLC cells treated with NC were arrested at the $\mathrm{G} 2 / \mathrm{M}$ phase. As $\mathrm{NC}$ concentrations increased, the proportion of block at the G2/M phase gradually increased (Fig. 4). Compared with the control groups, the average proportion of G2/M phase for A549 cells at the concentration of $4 \mu \mathrm{M}$ was increased, and the average proportion of G2/M phase for $\mathrm{H} 1975$ cells in at the concentration of $32 \mu \mathrm{M}$ was increased (Fig. 4).

\section{NC increased ROS level}

There were 10000 cells collected in each group. As shown in Fig. 5a, b, NC could significantly enhance the level of ROS species. Increased levels of intracellular ROS were concentration-dependent. Compared with H1975 cells, the response of A549 cells to NC was more sensitive. When treated with about $50 \%$ inhibiting concentration of $\mathrm{NC}$, the level of ROS in A549 and H1975 cells was about 47.4 times higher and 3.74 times higher than that of the control, respectively (Fig. 5a, b). 

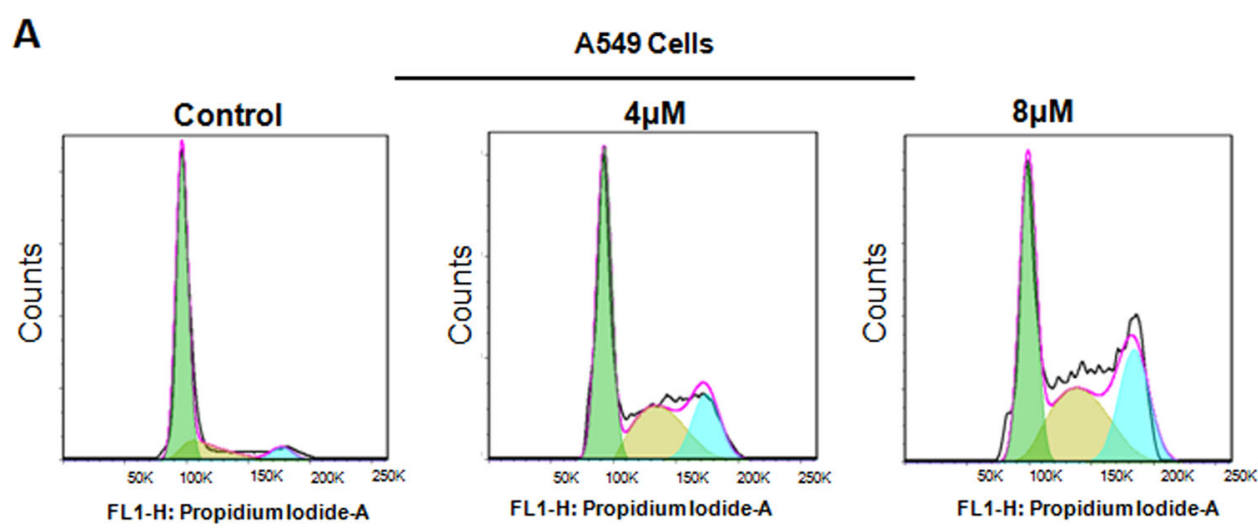

H1975 Cells
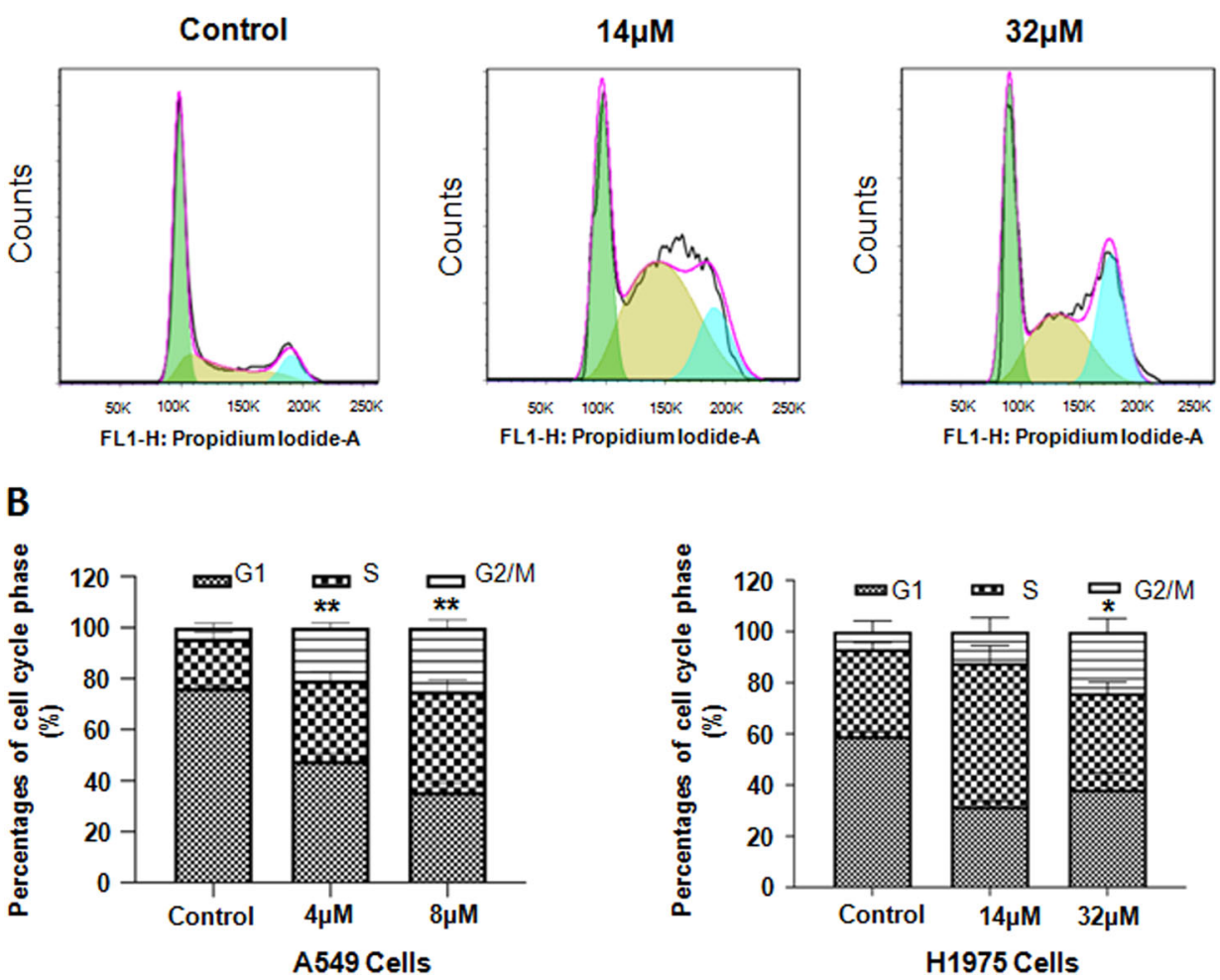

Fig. 4 NC caused cell cycle arrest of NSCLC cells. a Cell cycle in A549 and H1975 cells was detected by PI staining after treating with different doses of NC for $48 \mathrm{~h}$ by flow cytometry. $\mathbf{b}$ The percentage of each cell cycle phase of A549 and H1975 cells was illustrated. ${ }^{*} P<0.05$, ${ }^{* *} P<0.01$ vs. control.

\section{NC activated the Hippo signaling pathway}

Previous evidence indicates that the Hippo pathway is associated with the occurrence and development of NSCLC. However, it was not clear whether NC affected NSCLC cells through Hippo pathway. To test this possibility, the expression of key proteins in the Hippo pathway was detected after $48 \mathrm{~h}$ treatment by NC. As depicted in Fig. 6a, b, the levels of MOB1, LATS1, and YAP were markedly reduced under NC treatment. Meanwhile, compared with control, NC treatment significantly enhanced the levels of p-MOB1, p-LATS1, p-YAP1 (ser127). These findings indicate NC could activate the Hippo pathway.

\section{Discussion}

In recent years, research on Hippo pathways in tumor development has been deepened. It was reported that the Hippo signaling pathway is related to the progression of lung cancer. Studies show that YAP1 mutation leads to higher susceptibility to lung adenocarcinoma ${ }^{22}$. YAP is an 

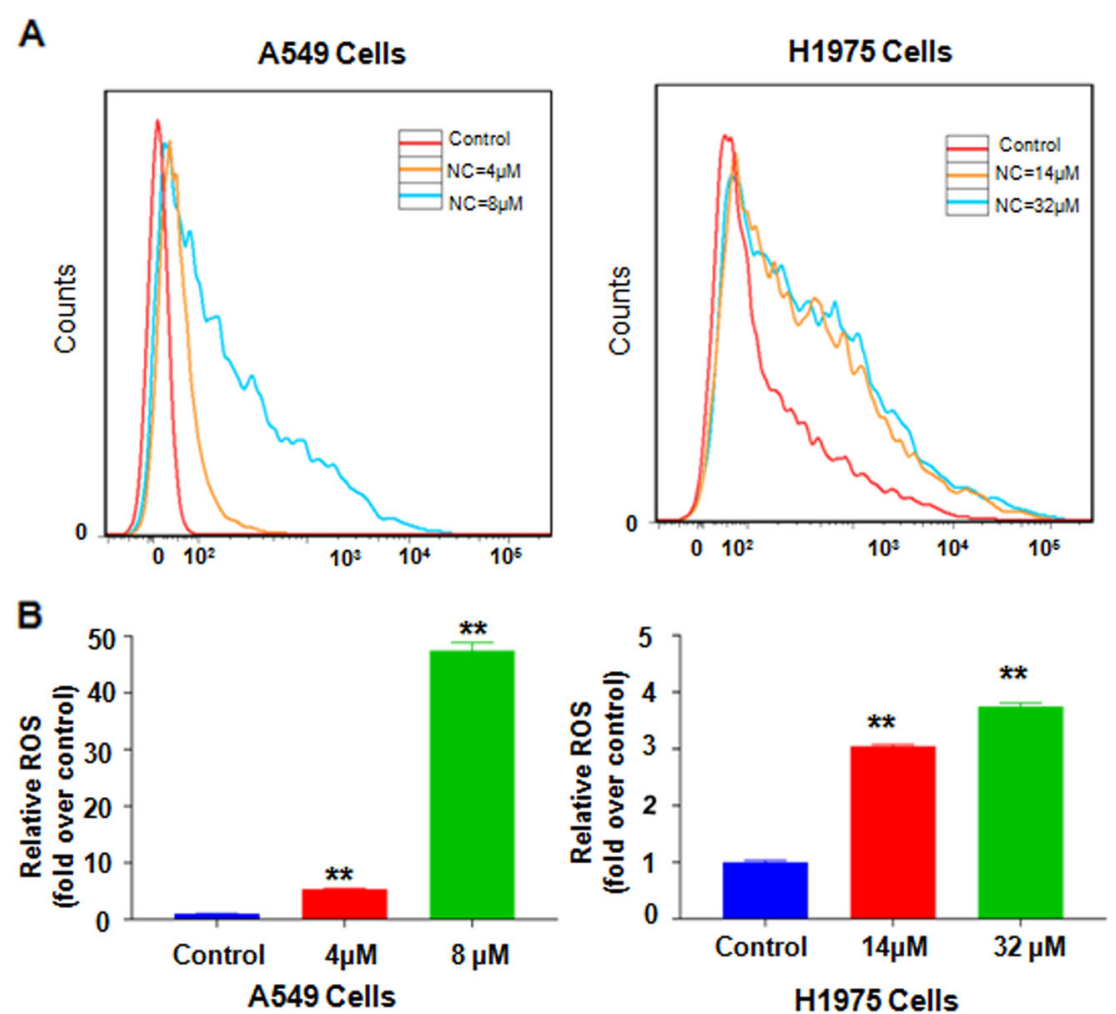

Fig. 5 NC promoted ROS level in NSCLC cells. a ROS level in A549 and H1975 cells was detected by DCFH-DA staining after treating with different doses of NC for $48 \mathrm{~h}$ by flow cytometry. $\mathbf{b}$ The percentage of relative ROS compared with control in A549 and H1975 cells was illustrated. ${ }^{* * P}<0.01$ vs. control.

important factor in the development and progression of lung cancer in mice $^{23}$. Lau et al. found that the proliferation and metastasis of lung tumor cells were associated with YAP/TAZ, and YAP activation will affect the progress of lung tumors in vivo ${ }^{24}$. YAP inhibition reduced brain metastasis of lung adenocarcinoma cells in nude mice $^{25}$. Interestingly, Hippo pathway is involved in the regulation of tumor immunogenicity ${ }^{26}$. The transcription of immune checkpoint PD-L1 was controlled by YAP in lung cancer ${ }^{27,28}$. Inhibition of PD-L1 can reduce the proliferation and wound healing of EGFR-TKI-resistant lung adenocarcinoma cells ${ }^{28}$. Therefore, these reports indicated that Hippo signaling pathway may provide new clues for lung cancer therapy.

Growing body of researches has been suggested that the increased ROS contributed to carcinogenesis, leading to activation of cell growth and survival. Furthermore, exaggerated generation of ROS in cancer cells trigged cell death, resulting in anti-tumor effects ${ }^{29,30}$. Some evidence report that oxidative stress regulates the Hippo pathway in cancer cells ${ }^{31}$. A recent study revealed that in malignant melanoma cells, ROS might be required for MST1 activation $^{32}$. In gastric cancer cells, the production of ROS was significantly related to the anti-tumor activity induced by the Curcumin Analogue WZ35 and the down-regulation of $\mathrm{YAP}^{33}$. Inhibiting YAP can induce the accumulation of ROS, which helped to overcome the resistance to chemotherapy of hepatocellular carcinoma $(\mathrm{HCC})^{34}$. Thus, our data demonstrated that $\mathrm{NC}$ could obviously increase ROS production in lung cancer cells, which leads to enhanced tumor suppression function of NC.

Natural products have gained extensive attention as potential anticancer drugs for more effective biological activities and low cytotoxicity. In previous studies, we revealed that $\mathrm{NC}$ can be used as a YAP inhibitor to treat prostate cancer cells ${ }^{20}$. In the present study, our data demonstrated NC inhibited cell viability, motility, caused cell cycle arrest, and promoted apoptosis and ROS level in NSCLC cells. We further explored the potential mechanism which NC affected NSCLC cells. Our findings indicated that $\mathrm{NC}$ could activate the Hippo pathway. All these data indicated that the Hippo pathway participates in the process of NC treatment in NSCLC cells.

\section{Materials and methods}

Cell culture and NC treatment

A549 and H1975 were provided by Chinese Academy of Science Cell Bank (Shanghai, China). RPMI-1640 medium, 


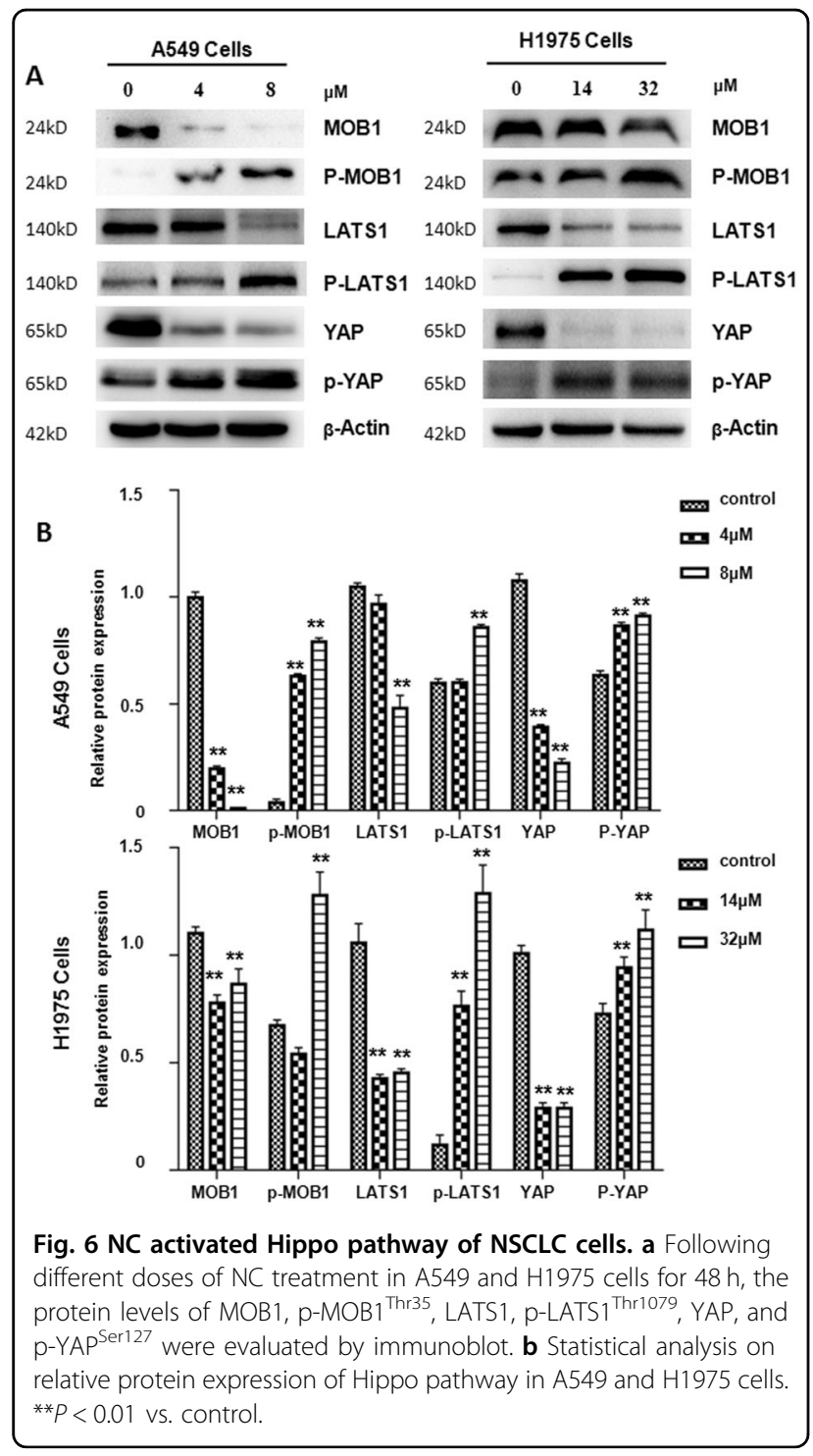

supplemented with 10\% FBS and 1\% penicillin-streptomycin mixture was used to culture cell lines. The cells were incubated in tissue culture dishes at $37^{\circ} \mathrm{C}$ in $5 \% \mathrm{CO}_{2}$ atmosphere. Nitidine chloride (NC, CAS no. 13063-04-2) was provided by Tauto Biotech Co. Ltd. (Shanghai, China). In order to guarantee the final concentration of dimethyl sulfoxide (DMSO) was $0.1 \%$, NC was firstly dissolved in DMSO as $25 \mathrm{mM}$ stocks, respectively, and stored at $-20^{\circ} \mathrm{C}$. And then diluting it with RPMI-1640 medium to different concentrations of $\mathrm{NC}$, and $0.1 \%$ DMSO was maintained in all samples.

\section{Reagents and antibodies}

Reactive oxygen species (ROS) Assay Kit and Cell Counting Kit-8 (CCK-8) assay were provided by Beyotime Institute of Biotechnology (Shanghai, China). Matrigel and FITC Annexin V Apoptosis Detection Kit I (\#556547) were provided by BD Biosciences (Franklin Lakes, NJ,
USA). The secondary anti-mouse and anti-rabbit antibodies were provided by Cell Signaling Technology (Danvers, MA, USA). Transwell plates were obtained from Corning Incorporated (Corning, NY, USA).

\section{CCK-8 assay}

Cell viability was detected by the CCK-8 assay. A549 or H1975 cells were seeded in a plate. After the cells were attached, each well was treated with NC or DMSO (vehicle control) and incubated for 48 and $72 \mathrm{~h}$ at specified concentrations. Each well was treated with $15 \mu \mathrm{l}$ CCK-8 solution and incubated for $2 \mathrm{~h}$. Measure absorption at $450 \mathrm{~nm}$ with a multi-functional microplate reader (BioTek, cytation3, USA).

\section{Colony formation assay}

For each well, $1.5 \times 10^{3}$ cell were seeded in 6-well-plate and incubated overnight, $\mathrm{NC}$ and DMSO were added to each well for 7 days in a humidified $5 \% \mathrm{CO}_{2}$ atmosphere at $37^{\circ} \mathrm{C}$. Before the cells were fixed with $4 \%$ paraformaldehyde for $20 \mathrm{~min}$, PBS was selected to wash cells for twice, and added crystal violet solution for staining and rinsed under running water. The visible colonies were counted by Imagej software.

\section{Transwell assay}

In order to detect the capacity of NSCLC cells to invade and migrate, cells are planted in the transwell chamber according to the manufacturer protocol. Compared with migration assay, invasion assay is different in that the polycarbonate membrane is coated with $60 \mu \mathrm{l}$ of $1 \mathrm{mg} / \mathrm{ml}$ matrigel for $2 \mathrm{~h}$. The cells treated with NC or DMSO were harvested and suspended in RPMI-1640 medium without serum. Then, $4 \times 10^{4}$ cells were transplanted into the upper inserts, and $700 \mu \mathrm{l}$ completed RPMI-1640 medium was injected into lower chamber. After $24 \mathrm{~h}$, cotton swabs were selected to remove non-migration or non-invasive cells. The cells which reached the lower surface through coated membranes were fixed by $4 \%$ PAF and stained by Crystal Violet solution. The invaded cells were photographed with a microscope, and counted at least six randomly-selected images to represent the relative migration and invasion.

\section{Cell apoptosis assay}

A549 or H1975 cells were seeded in 6-well plates and treated with NC or DMSO for $48 \mathrm{~h}$ after the cells adhered. Cells were digested by trypsin without ethylenediaminetetaacetic acid (EDTA) and washed with pre-cooled PBS. The collected cells were suspended in $100 \mu \mathrm{L}$ binding buffer $(1 \times)$, then incubated with $5 \mu \mathrm{L}$ FITC Annexin V and $5 \mu \mathrm{L}$ PI for $15 \mathrm{~min}$ in the dark $\left(25^{\circ} \mathrm{C}\right)$, and then added $400 \mu \mathrm{L}$ binding buffer $(1 \times)$. Finally, the stained cells were measured within $1 \mathrm{~h}$ of staining by a FACSVerse flow 
cytometer (BD Biosciences, USA), then the measurement results of assay was analyzed by FlowJo software (version7.6.1; Treestar, USA).

\section{Cell cycle analysis assay}

For determining the effect of NC on cell cycle, A549 or H1975 cells were cultivated in a 6-well-plate, used serumfree RPMI-1640 medium to grow to $70 \%$ confluency. Removed the serum-free medium and treated the cells with NC, respectively. After $48 \mathrm{~h}$ at $37^{\circ} \mathrm{C}$, the cells were digested by trypsin without EDTA, collected by centrifugation at $2000 \mathrm{rpm}$ for $5 \mathrm{~min}$, and then were fixed in cold ethanol for $24 \mathrm{~h}$ at $4{ }^{\circ} \mathrm{C}$. The cells were washed with pre-chilled PBS and incubated with a propidium iodide solution containing RNaseA $(0.1 \mathrm{~g} / \mathrm{mL})$ for $30 \mathrm{~min}$ in the dark at $37{ }^{\circ} \mathrm{C}$. After cells were filtered through a nylon mesh $(45-\mu \mathrm{m}$ well), the distribution of cell cycle was processed by using flow cytometry. Finally, the measurement data were analyzed by Flowjo software. The difference among multiple groups was analyzed by one-way analysis of variance (ANOVA).

\section{ROS level assay}

ROS in cells oxidized DCFH to generate fluorescent DCF which was then detected. Firstly, NSCLC cells were cultivated in a 6-well plate, and tests were carried out at different concentrations of NC in different groups. The cells in different sample-plates were collected respectively by trypsin digestion and centrifugation, incubated and stained with DCFH-DA for $30 \mathrm{~min}$ at $37^{\circ} \mathrm{C}$. Then, the samples were filtered through a nylon mesh $(45-\mu \mathrm{m}$ well) before it was analyzed by flow cytometry.

\section{Immunoblotting}

A549 or H1975 cells treated with NC or DMSO were harvested and lysed to extract total protein from the cells in a cell lysis buffer supplemented with protease inhibitors. Then quantified by BCA protein assay, isocratic protein $(60-80 \mu \mathrm{g})$ from cell lysate was separated by $10 \%$ SDS-PAGE and transferred to Immobilon ${ }^{\circledR}-\mathrm{P}$ Transfer Membrane. The membrane was incubated with indicated primary antibody. The primary antibodies were obtained from Cell Signaling Technology Company, including pYAP(Ser127, D9W2I, \#13008, 1:1000), MOB1 (E1N9D, \#13730, 1:1000), p-MOB1 (Thr35, D2F10, \#8699, 1:1000), LATS1(C66B5, \#3477, 1:1000), p-LATS1 (Thr1079, D57D3, \#8654, 1:1000), YAP (\#4912, 1:1000). The antiactin (A1978, 1:5000) antibody was bought from SigmaAldrich. Tris buffered saline, containing Tween 20 (TBST), was selected to wash the membrane and then the membrane was applied to the secondary antibody was incubated for $2 \mathrm{~h}\left(25^{\circ} \mathrm{C}\right)$. Finally, the chemiluminescent HRP substrate (Millipore Corpration, Billerica, MA, USA) was used and the immunoreactive bands were visualized by the Tanon5200 Detection System (Shanghai, China). ImageJ software was selected to analyze the protein expression intensity.

\section{Statistical analysis}

All data were showed as mean \pm standard deviation (SD). All data are representative of three independent experiments. All statistical analyses were performed by GraphPad Prism 6.0 software, and the data therein were expressed as mean \pm standard deviation (SD). Comparison of multiple groups was checked by ANOVA. $P<0.05$ and $P<0.01$ indicate that the difference is statistically significant.

\section{Acknowledgements}

The authors' research was supported in part by the major Project from the Natural Science Foundation of Education Department of Anhui Province (KJ2019ZD27), the Key Project of Natural Science Foundation of Anhui Provincial Department of Education (KJ2018A0221 and KJ2019A0354) and Anhui Provincial Undergraduate Innovative Training Program (S201910367041).

\section{Author details}

'Department of Genetics, School of Life Sciences, Bengbu Medical College, Anhui 233030, China. ${ }^{2}$ Bengbu Medical College Key Laboratory of Cancer Research and Clinical Laboratory Diagnosis, Bengbu Medical College, Anhui 233030, China. ${ }^{3}$ Department of Biochemistry and Molecular Biology, School of Laboratory Medicine, Bengbu Medical College, Anhui 233030, China. ${ }^{4}$ School of Clinical Medicine, Bengbu Medical College, Anhui 233030, China. ${ }^{5}$ School of Pharmacy, Bengbu Medical College, Anhui 233030, China. 'SChool of Life Sciences, Bengbu Medical College, Anhui 233030, China. ${ }^{7}$ Department of Laboratory Medicine, School of Laboratory Medicine, Bengbu Medical College, Bengbu, Anhui 233030, China

\section{Conflict of interest}

The authors declare that they have no conflict of interest.

\section{Publisher's note}

Springer Nature remains neutral with regard to jurisdictional claims in published maps and institutional affiliations.

Received: 5 July 2020 Revised: 15 August 2020 Accepted: 2 September 2020

Published online: 19 September 2020

\footnotetext{
References

1. Torre, L. A., Siegel, R. L. \& Jemal, A. Lung cancer statistics. Adv. Exp. Med Biol. 893, 1-19 (2016)

2. Ettinger, D. S. et al. Non-small cell lung cancer, Version 5.2017, NCCN clinical practice guidelines in oncology. J. Natl Compr. Canc Netw. 15, 504-535 (2017).

3. Lazzari, C. et al. Historical Evolution of Second-Line Therapy in Non-Small Cell Lung Cancer. Front. Med. (Lausanne) 4, 4 (2017).

4. Lo Sardo, F., Strano, S. \& Blandino, G. YAP and TAZ in lung cancer: oncogenic role and clinical targeting. Cancers (Basel) 10, 137 (2018).

5. Meng, Z., Moroishi, T. \& Guan, K. L. Mechanisms of Hippo pathway regulation. Genes Dev. 30, 1-17 (2016).

6. Ma, S., Meng, Z., Chen, R. \& Guan, K. L. The Hippo pathway: biology and pathophysiology. Annu. Rev. Biochem. 88, 577-604 (2019).

7. Zhao, B. et al. Inactivation of YAP oncoprotein by the Hippo pathway is involved in cell contact inhibition and tissue growth control. Genes Dev. 21, 2747-2761 (2007).

8. Hong, A. W., Meng, Z. \& Guan, K. L. The Hippo pathway in intestinal regeneration and disease. Nat. Rev. Gastroenterol. Hepatol. 13, 324-337 (2016).
} 
9. Pan, D. The hippo signaling pathway in development and cancer. Dev. Cell 19, 491-505 (2010).

10. Yu, F. X., Zhao, B. \& Guan, K. L. Hippo pathway in organ size control, tissue homeostasis, and cancer. Cell 163, 811-828 (2015).

11. Moroishi, T., Hansen, C. G. \& Guan, K. L. The emerging roles of YAP and TAZ in cancer. Nat. Rev. Cancer 15, 73-79 (2015).

12. Panciera, T., Azzolin, L., Cordenonsi, M. \& Piccolo, S. Mechanobiology of YAP and TAZ in physiology and disease. Nat. Rev. Mol. Cell Biol. 18, 758-770 (2017).

13. $\mathrm{Hu}$, J. et al. Benzophenanthridine alkaloids from Zanthoxylum nitidum (Roxb.) DC, and their analgesic and anti-inflammatory activities. Chem. Biodivers. 3 , 990-995 (2006).

14. Del Poeta, M. et al. Comparison of in vitro activities of camptothecin and nitidine derivatives against fungal and cancer cells. Antimicrob. Agents Chemother. 43, 2862-2868 (1999).

15. Wang, Z., Jiang, W., Zhang, Z., Qian, M. \& Du, B. Nitidine chloride inhibits LPSinduced inflammatory cytokines production via MAPK and NF-kappaB pathway in RAW 264.7 cells. J. Ethnopharmacol. 144, 145-150 (2012).

16. Mou, $\mathrm{H}$. et al. Nitidine chloride inhibited the expression of $\mathrm{S}$ phase kinaseassociated protein 2 in ovarian cancer cells. Cell Cycle 16, 1366-1375 (2017).

17. Zhai, $\mathrm{H}$. et al. Nitidine chloride inhibits proliferation and induces apoptosis in colorectal cancer cells by suppressing the ERK signaling pathway. Mol. Med Rep. 13, 2536-2542 (2016).

18. Lin, J. et al. Nitidine chloride inhibits hepatic cancer growth via modulation of multiple signaling pathways. BMC Cancer 14, 729 (2014).

19. Cui, Y. et al. Antitumor functions and mechanisms of nitidine chloride in human cancers. J. Cancer 11, 1250-1256 (2020).

20. Shi, Y. et al. Nitidine Chloride inhibits cell proliferation and invasion via downregulation of YAP expression in prostate cancer cells. Am. J. Transl. Res 11, 709-720 (2019).

21. $\mathrm{Xu}, \mathrm{H}$. et al. Nitidine Chloride Inhibits SIN1 Expression in Osteosarcoma Cells. Mol. Ther. Oncolytics 12, 224-234 (2019).

22. Chen, H. Y. et al. R331W Missense mutation of oncogene YAP1 is a germline risk allele for lung adenocarcinoma with medical actionability. J. Clin. Oncol. 33, 2303-2310 (2015).
23. Zhang, W. et al. YAP promotes malignant progression of Lkb1-deficient lung adenocarcinoma through downstream regulation of survivin. Cancer Res. 75, 4450-4457 (2015)

24. Lau, A. N. et al. Tumor-propagating cells and Yap/Taz activity contribute to lung tumor progression and metastasis. EMBO J. 33, 468-481 (2014).

25. Hsu, P. C. et al. Inhibition of yes-associated protein suppresses brain metastasis of human lung adenocarcinoma in a murine model. J. Cell Mol. Med. 22, 3073-3085 (2018).

26. Janse van Rensburg, H. J. et al. The Hippo pathway component TAZ promotes immune evasion in human cancer through PD-L1. Cancer Res. 78, 1457-1470 (2018).

27. Hsu, P. C., Jablons, D. M., Yang, C. T. \& You, L. Epidermal growth factor receptor (EGFR) pathway, Yes-associated protein (YAP) and the regulation of programmed death-ligand 1 (PD-L1) in non-small cell lung cancer (NSCLC). Int. J. Mol. Sci. 20, 3821 (2019)

28. Lee, B. S. et al. Hippo effector YAP directly regulates the expression of PD-L1 transcripts in EGFR-TKI-resistant lung adenocarcinoma. Biochem. Biophys. Res. Commun. 491, 493-499 (2017).

29. Moloney, J. N. \& Cotter, T. G. ROS signalling in the biology of cancer. Semin. Cell Dev. Biol. 80, 50-64 (2018).

30. Firczuk, M. et al. Harnessing altered oxidative metabolism in cancer by augmented prooxidant therapy. Cancer Lett. 471, 1-11 (2020).

31. Ashraf, A. \& Pervaiz, S. Hippo circuitry and the redox modulation of hippo components in cancer cell fate decisions. Int.J. Biochem. Cell Biol. 69, 20-28 (2015).

32. Yu, T., Ji, J. \& Guo, Y. L. MST1 activation by curcumin mediates JNK activation, Foxo3a nuclear translocation and apoptosis in melanoma cells. Biochem. Biophys. Res Commun. 441, 53-58 (2013).

33. Chen, T. et al. The curcumin analogue WZ35 affects glycolysis inhibition of gastric cancer cells through ROS-YAP-JNK pathway. Food Chem. Toxicol. 137 111131 (2020).

34. Zhou, Y. et al. YAP promotes multi-drug resistance and inhibits autophagyrelated cell death in hepatocellular carcinoma via the RAC1-ROS-mTOR pathway. Cancer Cell Int. 19, 179 (2019). 